\title{
First observation of the decay $B_{s}^{0} \rightarrow K_{\mathrm{S}}^{0} K^{*}(892)^{0}$ at $\mathrm{LHCb}$
}

\section{KHCh}

\section{The LHCb collaboration}

E-mail: marianna.fontana@cern.ch

ABSTRACT: A search for $B_{(s)}^{0} \rightarrow K_{S}^{0} K^{*}(892)^{0}$ decays is performed using $p p$ collision data, corresponding to an integrated luminosity of $1.0 \mathrm{fb}^{-1}$, collected with the LHCb detector at a centre-of-mass energy of $7 \mathrm{TeV}$. The $B_{s}^{0} \rightarrow K_{S}^{0} K^{*}(892)^{0}$ decay is observed for the first time, with a significance of 7.1 standard deviations. The branching fraction is measured to be

$$
\mathcal{B}\left(B_{s}^{0} \rightarrow \bar{K}^{0} K^{*}(892)^{0}\right)+\mathcal{B}\left(B_{s}^{0} \rightarrow K^{0} \bar{K}^{*}(892)^{0}\right)=(16.4 \pm 3.4 \pm 2.3) \times 10^{-6},
$$

where the first uncertainty is statistical and the second is systematic. No evidence is found for the decay $B^{0} \rightarrow K_{S}^{0} K^{*}(892)^{0}$ and an upper limit is set on the branching fraction, $\mathcal{B}\left(B^{0} \rightarrow \bar{K}^{0} K^{*}(892)^{0}\right)+\mathcal{B}\left(B^{0} \rightarrow K^{0} \bar{K}^{*}(892)^{0}\right)<0.96 \times 10^{-6}$, at $90 \%$ confidence level. All results are consistent with Standard Model predictions.

KEYwORDS: Hadron-Hadron scattering, B physics

ARXIV EPRINT: 1506.08634 


\section{Contents}

1 Introduction 1

2 Detector and simulation 2

3 Event selection $\quad 2$

4 Fit model $\quad 4$

5 Systematic uncertainties 5

$\begin{array}{llr}6 & \text { Summary and conclusion } & 8\end{array}$

$\begin{array}{ll}\text { The LHCb collaboration } & 12\end{array}$

\section{Introduction}

Violation of the combined charge-conjugation and parity symmetry $(C P)$ is one of the fundamental ingredients to explain a dynamical generation of the observed matter-antimatter asymmetry in the universe [1]. In the Standard Model of particle physics (SM), $C P$ violation in the quark sector is generated by a single complex phase in the Cabibbo-KobayashiMaskawa matrix [2, 3]. However, the observed baryon asymmetry is too large to be explained by the SM mechanism alone [4]. Non-leptonic $B$ meson decays dominated by amplitudes involving a quark and a $W$ boson in a loop (penguin) are sensitive to the presence of non-SM physics processes. These processes could provide additional sources of $C P$ violation that might explain the observed baryon asymmetry. The $B_{(s)}^{0} \rightarrow K_{\mathrm{S}}^{0} h^{ \pm} h^{\prime} \mp$ $\left(h, h^{\prime}=\pi, K\right)$ decays are interesting for $C P$ violation measurements. ${ }^{1}$ Knowledge of the branching fractions of the various sub-modes, as reported in this paper, is an important input to the theory of $C P$-violation, particularly models of new-physics contributions to $b \rightarrow s$ transitions ref. [5] . The measurements also allow tests of QCD models (see, for example, the predictions in refs. [6-8]). If sufficient data are available, a common approach for three-body decays is to perform an amplitude analysis by studying the structure of the Dalitz plot [9]. If data are less abundant and the decay products originate from intermediate resonances, as in the present analysis, a quasi two-body approach can be used.

The LHCb collaboration has provided results for inclusive $B_{(s)}^{0} \rightarrow K_{\mathrm{S}}^{0} h^{ \pm} h^{\prime} \mp$ decays [10], and more recently the first measurements of $B_{s}^{0}$ meson decays to $K^{*}(892)^{-} \pi^{+}$and $K^{*}(892)^{-} K^{+}$final states [11]. An initial search for the neutral decay $B^{0} \rightarrow K^{*}(892)^{0} K_{\mathrm{S}}^{0}$ has been reported by the BaBar experiment [12]. In this paper a search for $B_{(s)}^{0} \rightarrow$

\footnotetext{
${ }^{1}$ Charge-conjugate modes are implicitly included throughout this paper.
} 
$K_{\mathrm{S}}^{0} K^{*}(892)^{0}$ decays is reported, where the $K^{*}(892)^{0}$ meson, hereafter denoted by $K^{* 0}$, decays to the $K^{+} \pi^{-}$final state. The resonant structure in the $K^{+} \pi^{-}$invariant mass region around $1 \mathrm{GeV} / c^{2}$ is analysed to determine the number of decays that proceed through an intermediate $K^{* 0}$ resonance. The branching fraction is measured relative to the $B^{0} \rightarrow K_{\mathrm{S}}^{0} \pi^{+} \pi^{-}$decay [13], using the relation

$$
\frac{\mathcal{B}\left(B_{(s)}^{0} \rightarrow K_{\mathrm{S}}^{0} K^{* 0}\right)}{\mathcal{B}\left(B^{0} \rightarrow K_{\mathrm{S}}^{0} \pi^{+} \pi^{-}\right)}=\frac{N_{B_{(s)}^{0} \rightarrow K_{\mathrm{S}}^{0} K^{* 0}}}{N_{B^{0} \rightarrow K_{\mathrm{S}}^{0} \pi^{+} \pi^{-}}} \cdot \frac{\epsilon_{B^{0} \rightarrow K_{\mathrm{S}}^{0} \pi^{+} \pi^{-}}}{\epsilon_{B_{(s)}^{0} \rightarrow K_{\mathrm{S}}^{0} K^{* 0}}} \cdot \frac{f_{d}}{f_{d(s)}} \cdot \frac{1}{\mathcal{B}\left(K^{* 0} \rightarrow K^{+} \pi^{-}\right)},
$$

where $N$ represents the number of observed decays, $\epsilon$ the total efficiency, and $f_{s} / f_{d}$ the ratio of the fragmentation fractions of a $b$ quark into a $B_{s}^{0}$ or a $B^{0}$ meson [14-16] and $\mathcal{B}\left(K^{* 0} \rightarrow K^{+} \pi^{-}\right)$is the branching fraction of the $K^{* 0}$ meson into $K^{+} \pi^{-}$final state. In the following, the $B_{(s)}^{0} \rightarrow K_{\mathrm{S}}^{0} K^{* 0}$ and $B^{0} \rightarrow K_{\mathrm{S}}^{0} \pi^{+} \pi^{-}$decays are referred to as signal and normalisation channels, respectively.

\section{Detector and simulation}

The analysis is performed using $p p$ collision data recorded with the LHCb detector, corresponding to an integrated luminosity of $1.0 \mathrm{fb}^{-1}$, at a centre-of-mass energy of $7 \mathrm{TeV}$. The LHCb detector $[17,18]$ is a single-arm forward spectrometer covering the pseudorapidity range $2<\eta<5$, designed for the study of particles containing $b$ or $c$ quarks. The detector includes a high-precision tracking system consisting of a silicon-strip vertex detector surrounding the $p p$ interaction region, a large-area silicon-strip detector located upstream of a dipole magnet with a bending power of about $4 \mathrm{Tm}$, and three stations of silicon-strip detectors and straw drift tubes placed downstream of the magnet. The tracking system provides a measurement of momentum, $p$, of charged particles with a relative uncertainty that varies from $0.5 \%$ at low momentum to $1.0 \%$ at $200 \mathrm{GeV} / c$. The minimum distance of a track to a primary vertex $(\mathrm{PV})$, the impact parameter, is measured with a resolution of $\left(15+29 / p_{\mathrm{T}}\right) \mu \mathrm{m}$, where $p_{\mathrm{T}}$ is the component of the momentum transverse to the beam, in $\mathrm{GeV} / c$. Different types of charged hadrons are distinguished using information from two ring-imaging Cherenkov detectors. Photons, electrons and hadrons are identified by a calorimeter system consisting of scintillating-pad and preshower detectors, an electromagnetic calorimeter and a hadronic calorimeter. Muons are identified by a system composed of alternating layers of iron and multiwire proportional chambers.

Simulated events are used to determine the efficiency of the selection requirements, to study possible sources of background and to determine the parametrisations used to model the data. In the simulation, pp collisions are generated using Pутніа 6 [19] with a specific LHCb configuration [20]. Decays of hadronic particles are described by EvTGEN [21], in which final-state radiation is generated using Pнотоs [22]. The interaction of the generated particles with the detector, and its response, are implemented using the GEANT4 toolkit $[23,24]$ as described in ref. [25].

\section{Event selection}

The online event selection system (trigger) [26] consists of a hardware stage, based on information from the calorimeter and muon systems, followed by a software stage, in which 
all charged particles with $p_{\mathrm{T}}>500 \mathrm{MeV} / c$ are reconstructed. The hardware hadron trigger requires a calorimeter cluster with transverse energy greater than $3.5 \mathrm{GeV}$. In the offline selection, candidates are divided into two non mutually exclusive categories based on the hardware trigger decision. One category consists of candidates whose decay products satisfy the hadron trigger requirements, while the other consists of candidates from events in which other particles meet the hardware trigger requirements. Only events that fall into either of these categories are used in the subsequent analysis. The software trigger requires a two-, three- or four-particle secondary vertex with a significant displacement from the primary $p p$ interaction points. At least one charged particle must have $p_{\mathrm{T}}>1.7 \mathrm{GeV} / c$ and be inconsistent with originating from any PV. A multivariate algorithm [27] is used for the identification of secondary vertices consistent with the decay of a $b$ hadron.

In the offline selection the $B_{(s)}^{0} \rightarrow K_{\mathrm{S}}^{0} K^{* 0}$ decays are reconstructed through the $K^{* 0}$ $\rightarrow K^{+} \pi^{-}$and $K_{\mathrm{S}}^{0} \rightarrow \pi^{+} \pi^{-}$decay modes, where the $K_{\mathrm{S}}^{0}$ candidate is constrained to its known mass [13] and the $B$ candidate is constrained to originate from a PV. Decays of $K_{\mathrm{S}}^{0}$ mesons are reconstructed in two mutually exclusive categories: long $K_{\mathrm{S}}^{0}$ candidates, which decay sufficiently early that their daughter pions are reconstructed in the vertex detector; and downstream $K_{\mathrm{S}}^{0}$ candidates, which have daughter particles that are only reconstructed in the rest of the tracking system. As these two categories have different backgrounds, and the long $K_{\mathrm{S}}^{0}$ mesons have better momentum and vertex resolutions, the selection criteria for long and downstream $K_{\mathrm{S}}^{0}$ candidates differ. The selection criteria follow those in ref. [10].

Fully reconstructed background decays that have the same final state as the signal include contributions from $B$ decays to final states involving charm mesons, such as $D h$, with a $K_{\mathrm{S}}^{0} h^{+} h^{-}$final state, or $\Lambda_{b}^{0}$ decays to $\Lambda_{c}^{+} h^{-}$, with $\Lambda_{c}^{+} \rightarrow K_{\mathrm{S}}^{0} p$, where the proton is misidentified as a $\pi^{+}$or $K^{+}$. In addition, $B$ decays with an intermediate charmonium state like $B^{0} \rightarrow J / \psi K_{\mathrm{S}}^{0}$, with $J / \psi \rightarrow \pi^{+} \pi^{-}, K^{+} K^{-}, \mu^{+} \mu^{-}$, can be present in the mass region of the normalisation channel. To reduce the contamination from these backgrounds, a veto is applied on the invariant mass of each of the possible intermediate states reconstructed under the corresponding hypothesis. Candidates are excluded if the reconstructed mass of a two-body intermediate state is within $30 \mathrm{MeV} / c^{2}\left(48 \mathrm{MeV} / c^{2}\right)$ of the known mass of the relevant intermediate charm (charmonium) resonance [13] of one of the backgrounds considered. No particle identification information is used at this stage.

If a final-state hadron is misidentified, signal yields can potentially be affected by decays into any $K_{\mathrm{S}}^{0} h^{ \pm} h^{\prime \mp}$ final state, especially when the $h^{ \pm} h^{\prime \mp}$ proceeds through a resonance. Particle identification requirements on the two tracks originating from the $B$ decay vertex are used to separate pions, kaons and protons, and to reduce this background to a negligible level. The largest source of background is due to random tracks that form candidate $B$ or $K_{\mathrm{S}}^{0}$ decay vertices. A multivariate discriminant based on a boosted decision tree (BDT) algorithm $[28,29]$ is used to reduce this background. The greatest discrimination in the BDT is provided by kinematic properties of the $B$ meson, its flight direction with respect to the PV, and variables defined analogously for its decay products. The optimisation of the BDT is described in ref. [10]; the selection requirement on the BDT response for this analysis is chosen to maximise $\epsilon /\left(a / 2+\sqrt{N_{B}}\right)$ [30]. Here, $\epsilon$ is the signal efficiency, $B$ represents the number of background events in the signal mass interval, which is estimated 
using data by extrapolating the number of background events from the upper mass sideband into the signal region, and $a=5$ is the chosen target signal significance.

The efficiencies are determined from simulation, except for the particle identification efficiencies. The latter are determined from data using samples of kinematically identified charged particles from $D^{*+} \rightarrow D^{0} \pi^{+}$with $D^{0} \rightarrow K^{-} \pi^{+}$, and $\Lambda \rightarrow \pi^{-} p$ decays, reweighted to match the kinematic properties of the signal. The BDT selection efficiency for signal is approximately $85 \%$ (90\%) for downstream (long) signal decays; approximately $88 \%$ $(95 \%)$ of backgrounds in the respective categories are rejected. The $B^{0} \rightarrow K_{\mathrm{S}}^{0} \pi^{+} \pi^{-}$decay selection efficiency is taken from ref. [10]. The efficiencies for the normalisation channel are determined in bins of the Dalitz plane and are reweighted from data using the sPlot method [31].

\section{$4 \quad$ Fit model}

Two-dimensional extended maximum likelihood fits to the unbinned $K_{\mathrm{S}}^{0} K^{+} \pi^{-}$and $K^{+} \pi^{-}$ mass distributions are used to determine the event yields for the signal channel, while an independent one-dimensional fit to the $K_{\mathrm{S}}^{0} \pi^{+} \pi^{-}$mass distribution is used for the normalisation channel. The correlation between the two signal mass distributions is checked on simulation. The results do not show significant correlations and therefore the correlation terms are neglected in the fit. Candidates in the long and downstream categories are fitted simultaneously. The signal fit is restricted to candidates in the mass regions $5000<$ $m\left(K_{\mathrm{S}}^{0} K^{+} \pi^{-}\right)<5800 \mathrm{MeV} / c^{2}$ and $650<m\left(K^{+} \pi^{-}\right)<1200 \mathrm{MeV} / c^{2}$. The fit model consists of signal, non-resonant background, partially reconstructed background and combinatorial background components.

The $B^{0}$ and the $B_{s}^{0}$ components of the signal are both parametrised as two Gaussian distributions with a power-law tail on each side. For each component the two functions share the peak position and the width parameters. The parameters describing the tails are determined by fits to simulated samples and subsequently fixed in the fit to data. The systematic uncertainty associated with this choice is found to be negligible. The $B^{0}-B_{s}^{0}$ mass difference is fixed to the known value [13]. The $K^{* 0}$ mass distribution is parametrised by a relativistic Breit-Wigner function with the peak position and width allowed to vary in the fit.

The components in the $B$ mass model that are non-resonant in $K^{+} \pi^{-}$are parametrised by the same function as the signal, sharing their peak positions and widths with the signal functions. The tail parameters are fixed according to the values obtained from simulation. The non-resonant component of the $K^{+} \pi^{-}$mass distribution is approximated by a normalised linear function as in ref. [11], with the zero point of the function on the abscissa determined by the fit. While the ratio between the non-resonant and the signal components is fixed to be the same for the two $K_{\mathrm{S}}^{0}$ meson categories, it is independent for the $B^{0}$ and the $B_{s}^{0}$ candidates.

Backgrounds from partially reconstructed decays are classified into two categories. Decays such as $B \rightarrow D h$ are parametrised by means of ARGUS functions convolved with a Gaussian function in the $B$ candidate mass, and linear functions in the $K^{* 0}$ candidate 


\begin{tabular}{|lrcrc|}
\hline Decay & \multicolumn{2}{c}{ Downstream } & \multicolumn{2}{c|}{ Long } \\
\cline { 2 - 5 } & \multicolumn{1}{c}{ Yield } & Efficiency (\%) & Yield & Efficiency (\%) \\
\hline$B_{s}^{0} \rightarrow K_{\mathrm{S}}^{0} K^{* 0}$ & $21 \pm 6$ & $0.0174 \pm 0.0012$ & $25 \pm 6$ & $0.0121 \pm 0.0008$ \\
$B^{0} \rightarrow K_{\mathrm{S}}^{0} K^{* 0}$ & $2 \pm 3$ & $0.0183 \pm 0.0013$ & $1 \pm 2$ & $0.0125 \pm 0.0009$ \\
$B^{0} \rightarrow K_{\mathrm{S}}^{0} \pi^{+} \pi^{-}$ & $828 \pm 41$ & $0.0336 \pm 0.0010$ & $341 \pm 23$ & $0.0117 \pm 0.0009$ \\
\hline
\end{tabular}

Table 1. Signal yields obtained from the fits to $K_{\mathrm{S}}^{0} K^{ \pm} \pi^{\mp}$ and $K_{\mathrm{S}}^{0} \pi^{+} \pi^{-}$mass distributions and corresponding efficiencies. Only statistical contributions to the uncertainty are reported.

mass. The choice is based on simulation studies and previous findings [10, 11]. In decays such as $B_{s}^{0} \rightarrow K^{* 0} \bar{K}^{* 0}$, where one resonance decays as $K^{* 0} \rightarrow K^{+} \pi^{-}$while the other decays as $\bar{K}^{* 0} \rightarrow K_{\mathrm{S}}^{0} \pi^{0}$, the $B_{s}^{0}$ mass distribution is described using the same parametrisation as for the previous background, while the invariant mass distribution for $K^{* 0}$ candidates is described by a relativistic Breit-Wigner function sharing the peak position and widths with the signal component. The yield for these components are determined in the fit to data.

The combinatorial background is modelled by an exponential function in the $B$ candidate mass distribution and a linear function in the $K^{* 0}$ candidate mass distribution. These functions are found to give good agreement with the distributions in the appropriate data sidebands. The slopes of the exponential functions are independent for the long and downstream categories, while the abscissae of the linear functions are the same. All these parameters are allowed to vary in the fit.

The parametrisation used to model the $B^{0} \rightarrow K_{\mathrm{S}}^{0} \pi^{+} \pi^{-}$normalisation and the background follow those used to fit the signal mode. In addition two other categories of partially reconstructed backgrounds are included: decays such as $B^{0} \rightarrow K_{\mathrm{S}}^{0} \pi^{+} \pi^{-} \gamma$ or $B^{0} \rightarrow \eta^{\prime} K_{\mathrm{S}}^{0}$, with $\eta \rightarrow \rho^{0} \gamma$; and misidentified $B_{(s)}^{0} \rightarrow K_{\mathrm{S}}^{0} K^{ \pm} \pi^{\mp}$ decays. Their parameters are fixed in the fit to the values derived from simulated samples.

The observed $K_{\mathrm{S}}^{0} K^{* 0}$ and $K_{\mathrm{S}}^{0} \pi^{+} \pi^{-}$mass distributions and the corresponding fits are shown in figures 1 and 2, respectively. The signal yields are reported in table 1 . The $B^{0}$ mode is dominated by the non-resonant component. The statistical significance of the $B_{s}^{0}$ signal is determined using Wilks' theorem [32] and by combining the long and the downstream samples. The significance including relevant systematic uncertainties, estimated by repeating the procedure with the signal likelihood convolved with a Gaussian function of width equal to the sum in quadrature of the systematic uncertainties, is 7.1 standard deviations.

\section{Systematic uncertainties}

The model used to fit data and the limited knowledge in the efficiency determination are possible sources of systematic uncertainty. Many parameters in the fit are fixed to values obtained from fits to simulated data. The associated systematic uncertainties are determined from fits to pseudoexperiments generated assuming alternative values of the relevant parameters, corresponding to variations within uncertainties around their default 

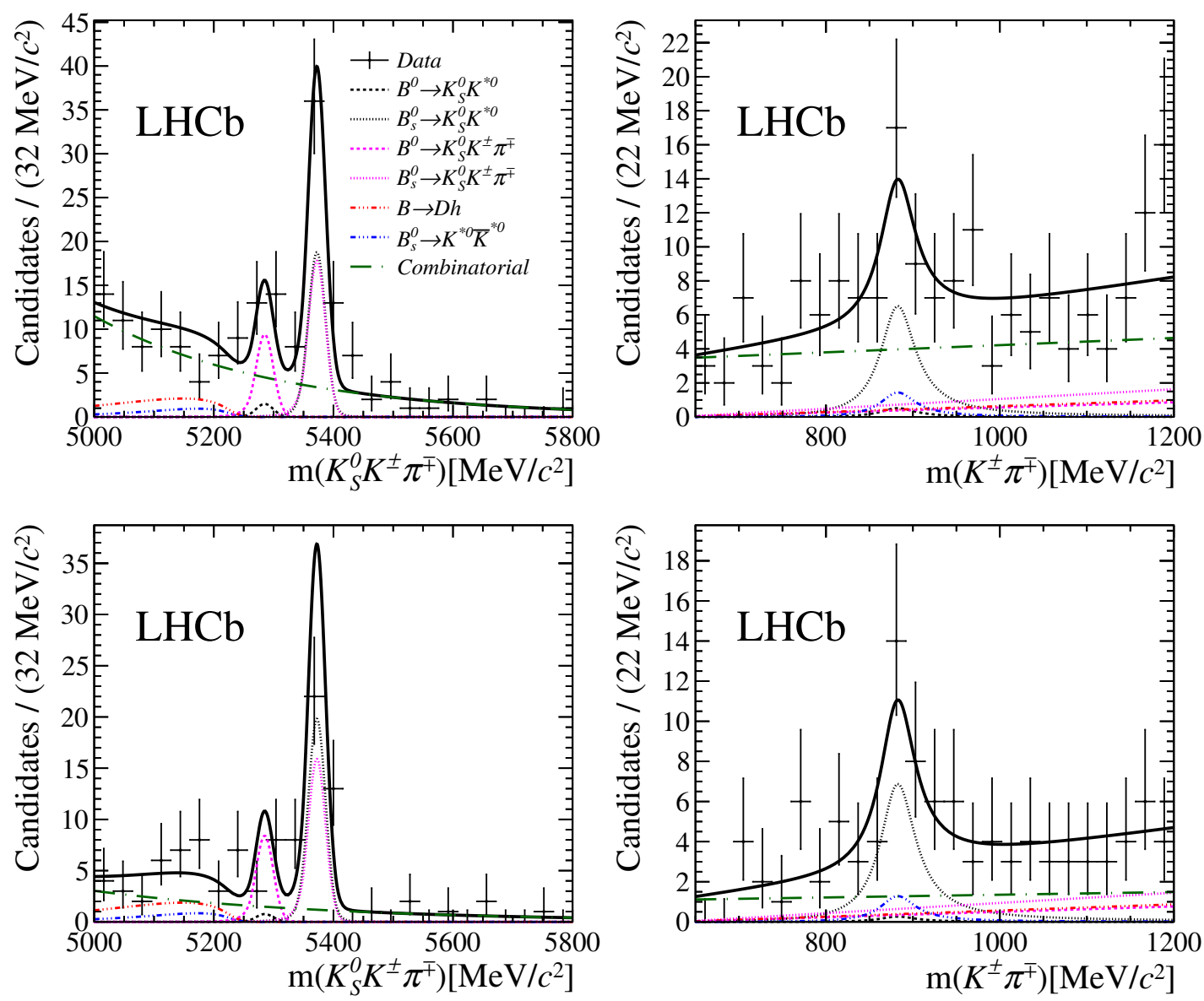

Figure 1. Distribution of (left) $K_{\mathrm{S}}^{0} K^{ \pm} \pi^{\mp}$ mass and (right) $K^{ \pm} \pi^{\mp}$ mass for signal candidates with fit results overlaid for (top) downstream and (bottom) long categories. The data are shown as black points with error bars. The overall fit is represented by the solid black line. The $B^{0}$ and $B_{s}^{0}$ signal components are the black short-dashed and dotted lines respectively, while the nonresonant components are the magenta short-dashed and dotted lines. The partially reconstructed backgrounds are the red triple-dotted line $(B \rightarrow D h)$ and the blue triple-dotted line $\left(B_{s}^{0} \rightarrow K^{* 0} \bar{K}^{* 0}\right)$. The combinatorial background is the green long-dash dotted line.

values. The average difference between the yields determined in the pseudoexperiments and the nominal value is taken as a systematic uncertainty.

The fit model does not account for the possible interference between the $\mathrm{P}$ wave of the $K^{*}(892)^{0}$ resonance and the $\mathrm{S}$ wave from other intermediate states, e.g. the nonresonant component or the $K^{*}(1430)^{0}$ resonance. The associated systematic uncertainty is determined by exploiting the distribution of $\theta_{K^{* 0}}$, defined as the angle between the flight direction of the $K^{+}$in the $K^{* 0}$ rest frame with respect to the direction of the boost from the laboratory frame to the $K^{* 0}$ rest frame. The $\cos \theta_{K^{* 0}}$ distribution is described by a parabola, where the second-order term represents the signal $\mathrm{P}$ wave, the constant term is related to the $\mathrm{S}$ wave and the first-order term accounts for the interference. Using the $s P l o t$ technique [31], the $\cos \theta_{K^{* 0}}$ distribution of the signal $\mathrm{P}$ and $\mathrm{S}$ wave is unfolded from the other background components. A fit in the region of positive $\cos \theta_{K^{* 0}}$ is performed using 

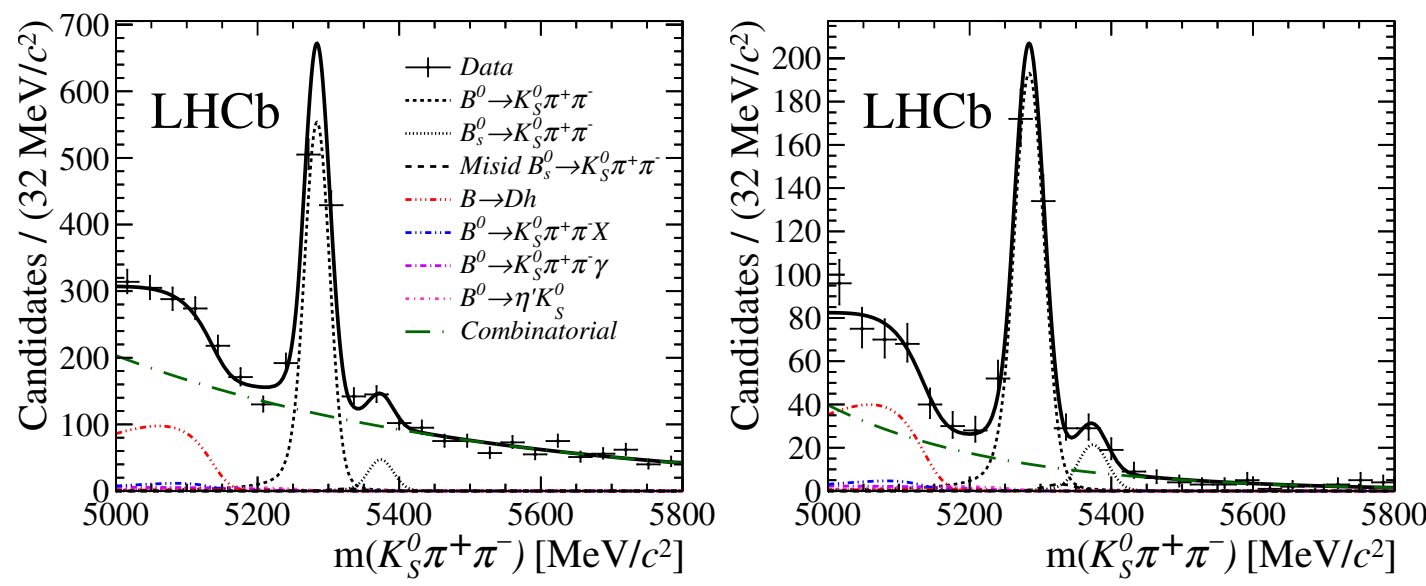

Figure 2. Distribution of $K_{\mathrm{s}}^{0} \pi^{+} \pi^{-}$mass for signal candidates with fit results overlaid for (left) downstream and (right) long categories. The data are described by the black points with error bars. The overall fit is represented by the solid black line. The $B^{0}$ and $B_{s}^{0}$ signal components are the black short-dashed and dotted lines. The misidentified $B_{s}^{0}$ decay is the black dashed line, respectively. The partially reconstructed backgrounds are the red triple-dotted line $(B \rightarrow D h)$, the blue triple-dotted line $\left(B^{0} \rightarrow K_{\mathrm{S}}^{0} \pi^{+} \pi^{-} X\right)$, the violet dash single-dotted line $\left(B^{0} \rightarrow \eta^{\prime} K_{\mathrm{S}}^{0}\right)$ and the pink short-dash single dotted line $\left(B^{0} \rightarrow K_{\mathrm{S}}^{0} \pi^{+} \pi^{-} \gamma\right)$. The combinatorial background is the green long-dash dotted line. Some of the contributions are small in the figures.

a second-order polynomial and the systematic uncertainty is determined as the relative difference between the integral of the function when the constant coefficient is allowed to vary and when this coefficient is fixed to zero. Due to the limited size of the $B^{0} \rightarrow K_{\mathrm{S}}^{0} K^{* 0}$ sample, the relative uncertainty obtained for the $B_{s}^{0} \rightarrow K_{\mathrm{S}}^{0} K^{* 0}$ decays is also applied to the $B^{0} \rightarrow K_{\mathrm{S}}^{0} K^{* 0}$ decays.

Potential biases that may be associated with the maximum likelihood estimator are investigated using pseudoexperiments. The systematic uncertainty is determined as the average difference between the nominal value and the fitted yields in the pseudoexperiments.

The impact of the limited size of the simulated samples, used to determine the selection and particle identification efficiencies, is considered as systematic uncertainty. In addition, the hardware trigger is a potential source of systematic uncertainty due to imperfections in the description of data by simulation. A data sample of $D^{*+} \rightarrow D^{0} \pi^{ \pm}$, with $D^{0} \rightarrow K^{-} \pi^{+}$, decays is used to characterise the trigger efficiencies of the pions and kaons, separated according to particle charge, as a function of the transverse energy of the associated cluster in the hadron calorimeter $[26,33]$. These data-driven calibration curves are used to weight simulated events in order to determine the efficiency of the hadron trigger.

The effective lifetimes of $B_{s}^{0}$ meson reconstructed in a particular decay depend on the $C P$-admixture of the final state because $C P$-even and $C P$-odd eigenstates may have different lifetimes [34]. Since the selection efficiency depends on decay time, this might lead to a source of uncertainty in the measurement. The relative change in efficiency with respect to the nominal value, estimated for the extreme ranges of possible effective lifetime distributions, is assigned as the systematic uncertainty. 


\begin{tabular}{|lcccc|}
\hline \multirow{2}{*}{ Source } & \multicolumn{2}{c}{$\frac{\mathcal{B}\left(B_{S}^{0} \rightarrow K_{\mathrm{S}}^{0} K^{* 0}\right)}{\mathcal{B}\left(B^{0} \rightarrow K_{\mathrm{S}}^{\mathrm{S}} \pi^{+} \pi^{-}\right)}$} & \multicolumn{2}{c|}{$\frac{\mathcal{B}\left(B^{0} \rightarrow K_{\mathrm{S}}^{0} K^{* 0}\right)}{\mathcal{B}\left(B^{0} \rightarrow K_{\mathrm{S}}^{0} \pi^{+} \pi^{-}\right)}$} \\
\cline { 2 - 5 } & Downstream & Long & Downstream & Long \\
\hline Fit & 0.05 & 0.03 & 0.20 & 0.28 \\
Selection efficiency & 0.08 & 0.10 & 0.08 & 0.11 \\
PID efficiency & 0.01 & 0.01 & 0.01 & 0.01 \\
Trigger & 0.07 & 0.07 & 0.02 & 0.09 \\
Lifetime & 0.05 & 0.05 & - & - \\
\hline Total & 0.13 & 0.14 & 0.22 & 0.31 \\
\hline$f_{s} / f_{d}$ & 0.06 & 0.06 & - & - \\
\hline
\end{tabular}

Table 2. Systematic uncertainties on the relative branching fraction measurement for the two $K_{\mathrm{S}}^{0}$ categories. The uncertainties are quoted as fractional contributions of the relative branching fraction and the total is the sum in quadrature of all contributions.

Finally, the uncertainty from the measurement of the fragmentation fractions ratio, $f_{s} / f_{d}[14-16]$, is taken into account. A summary of the relative uncertainties on the ratio of branching fractions is given in table 2. The final results reported in section 6 take into account correlations between the two samples; thus the systematic uncertainty for the combined measurement is reduced.

\section{Summary and conclusion}

A search for $B_{(s)}^{0} \rightarrow K_{\mathrm{S}}^{0} K^{* 0}$ decays is performed by the LHCb experiment using $p p$ data recorded at a centre-of-mass energy of $7 \mathrm{TeV}$, corresponding to an integrated luminosity of $1.0 \mathrm{fb}^{-1}$. The branching ratios are determined using the $B^{0} \rightarrow K_{\mathrm{S}}^{0} \pi^{+} \pi^{-}$decay as a normalisation mode. The measurements are performed separately for the downstream and long $K_{\mathrm{S}}^{0}$ categories and then combined following refs. [35, 36].

The $B_{s}^{0}$ decay is observed for the first time, with a total significance of 7.1 standard deviations. The relative branching fraction is

$$
\frac{\mathcal{B}\left(B_{s}^{0} \rightarrow K_{\mathrm{S}}^{0} K^{* 0}\right)}{\mathcal{B}\left(B^{0} \rightarrow K_{\mathrm{S}}^{0} \pi^{+} \pi^{-}\right)}=0.33 \pm 0.07 \text { (stat) } \pm 0.04 \text { (syst) } \pm 0.02\left(f_{s} / f_{d}\right) \text {. }
$$

For the $B^{0}$ decay, an upper limit at $90 \%$ (95\%) confidence level (CL) is determined. The likelihood function is convolved with a Gaussian function with standard deviation equal to the total systematic uncertainty, and the upper limit is taken to be the value of the relative branching fraction below which $90 \%$ (95\%) of the total integral of the likelihood function over non-negative branching ratio values is found. The central value and the upper limit on the relative branching fraction of the decay $B^{0} \rightarrow K_{\mathrm{S}}^{0} K^{* 0}$ are

$$
\begin{aligned}
\frac{\mathcal{B}\left(B^{0} \rightarrow K_{\mathrm{S}}^{0} K^{* 0}\right)}{\mathcal{B}\left(B^{0} \rightarrow K_{\mathrm{S}}^{0} \pi^{+} \pi^{-}\right)} & =0.005 \pm 0.007 \text { (stat) } \pm 0.001 \text { (syst) } \\
& <0.020(0.021) \text { at } 90 \%(95 \%) \mathrm{CL}
\end{aligned}
$$


The absolute branching fractions, calculated using the reference value of $\mathcal{B}\left(B^{0} \rightarrow\right.$ $\left.K^{0} \pi^{+} \pi^{-}\right)=(4.96 \pm 0.20) \times 10^{-5}[37]$, determined without using the correlated $\mathrm{LHCb}$ measurement. The results are expressed in terms of the sum of final states containing either $K^{0}$ or $\bar{K}^{0}$ mesons

$$
\begin{aligned}
\mathcal{B}\left(B_{s}^{0} \rightarrow \bar{K}^{0} K^{*}(892)^{0}\right)+\mathcal{B}\left(B_{s}^{0} \rightarrow K^{0} \bar{K}^{*}(892)^{0}\right) & =(16.4 \pm 3.4 \pm 1.9 \pm 1.0 \pm 0.7) \times 10^{-6} \\
\mathcal{B}\left(B^{0} \rightarrow \bar{K}^{0} K^{*}(892)^{0}\right)+\mathcal{B}\left(B^{0} \rightarrow K^{0} \bar{K}^{*}(892)^{0}\right) & =(0.25 \pm 0.34 \pm 0.05 \pm 0.01) \times 10^{-6} \\
& <0.96(1.04) \times 10^{-6} \text { at } 90 \%(95 \%) \mathrm{CL}
\end{aligned}
$$

where the first uncertainty is statistical, the second systematic, the third due to the ratio of the fragmentation fractions and the fourth due to the uncertainty on the branching fraction of the normalisation decay. These results are in agreement with theoretical predictions [6-8] and can be used to further constrain phenomenological models.

\section{Acknowledgments}

We express our gratitude to our colleagues in the CERN accelerator departments for the excellent performance of the LHC. We thank the technical and administrative staff at the LHCb institutes. We acknowledge support from CERN and from the national agencies: CAPES, CNPq, FAPERJ and FINEP (Brazil); NSFC (China); CNRS/IN2P3 (France); BMBF, DFG, HGF and MPG (Germany); INFN (Italy); FOM and NWO (The Netherlands); MNiSW and NCN (Poland); MEN/IFA (Romania); MinES and FANO (Russia); MinECo (Spain); SNSF and SER (Switzerland); NASU (Ukraine); STFC (United Kingdom); NSF (U.S.A.). The Tier1 computing centres are supported by IN2P3 (France), KIT and BMBF (Germany), INFN (Italy), NWO and SURF (The Netherlands), PIC (Spain), GridPP (United Kingdom). We are indebted to the communities behind the multiple open source software packages on which we depend. We are also thankful for the computing resources and the access to software R\&D tools provided by Yandex LLC (Russia). Individual groups or members have received support from EPLANET, Marie Skłodowska-Curie Actions and ERC (European Union), Conseil général de Haute-Savoie, Labex ENIGMASS and OCEVU, Région Auvergne (France), RFBR (Russia), XuntaGal and GENCAT (Spain), Royal Society and Royal Commission for the Exhibition of 1851 (United Kingdom). We acknowledge Ulrich Nierste from KIT (Germany) for his assistance on theoretical aspects of the analysis.

Open Access. This article is distributed under the terms of the Creative Commons Attribution License (CC-BY 4.0), which permits any use, distribution and reproduction in any medium, provided the original author(s) and source are credited.

\section{References}

[1] A. Sakharov, Violation of CP invariance, $C$ asymmetry, and baryon asymmetry of the Universe, J. Exp. Theor. Phys. Lett. 5 (1967) 24.

[2] N. Cabibbo, Unitary symmetry and leptonic decays, Phys. Rev. Lett. 10 (1963) 531 [INSPIRE]. 
[3] M. Kobayashi and T. Maskawa, CP violation in the renormalizable theory of weak interaction, Prog. Theor. Phys. 49 (1973) 652 [InSPIRE].

[4] A. Riotto and M. Trodden, Recent progress in baryogenesis, Ann. Rev. Nucl. Part. Sci. 49 (1999) 35 [hep-ph/9901362] [INSPIRE].

[5] M. Ciuchini, M. Pierini and L. Silvestrini, $B(s) \rightarrow K^{(*) 0} \bar{K}^{(*) 0}$ decays: the golden channels for new physics searches, Phys. Rev. Lett. 100 (2008) 031802 [hep-ph/0703137] [INSPIRE].

[6] H.-Y. Cheng and C.-K. Chua, QCD factorization for charmless hadronic $B_{s}$ decays revisited, Phys. Rev. D 80 (2009) 114026 [arXiv:0910.5237] [InSPIRE].

[7] A. Ali et al., Charmless non-leptonic $B_{s}$ decays to $P P, P V$ and $V V$ final states in the $p Q C D$ approach, Phys. Rev. D 76 (2007) 074018 [hep-ph/0703162] [INSPIRE].

[8] F. Su, Y.-L. Wu, C. Zhuang and Y.-B. Yang, Charmless $B_{s} \rightarrow P P, P V, V V$ decays based on the six-quark effective hamiltonian with strong phase effects II, Eur. Phys. J. C 72 (2012) 1914 [arXiv: 1107.0136] [INSPIRE].

[9] R. Dalitz, On the analysis of $\tau$-meson data and the nature of the $\tau$-meson, Phil.Mag. 44 (1953) 1068.

[10] LHCb collaboration, Study of $B_{(s)}^{0} \rightarrow K_{\mathrm{S}}^{0} h^{+} h^{\prime-}$ decays with first observation of $B_{s}^{0} \rightarrow K_{\mathrm{S}}^{0} K^{ \pm} \pi^{\mp}$ and $B_{s}^{0} \rightarrow K_{\mathrm{S}}^{0} \pi^{+} \pi^{-}$, JHEP 10 (2013) 143 [arXiv:1307.7648] [INSPIRE].

[11] LHCb collaboration, Observation of $B_{s}^{0} \rightarrow K^{* \pm} K^{\mp}$ and evidence for $B_{s}^{0} \rightarrow K^{*-} \pi^{+}$decays, New J. Phys. 16 (2014) 123001 [arXiv:1407.7704] [INSPIRE].

[12] BABAR collaboration, B. Aubert et al., Search for the decay of a $B^{0}$ or anti- $B^{0}$ meson to $\bar{K}^{* 0} K^{0}$ or $K^{* 0} \bar{K}^{0}$, Phys. Rev. D 74 (2006) 072008 [hep-ex/0606050] [INSPIRE].

[13] Particle Data Group collaboration, K.A. Olive et al., Review of particle physics, Chin. Phys. C 38 (2014) 090001 [INSPIRE].

[14] LHCb collaboration, Measurement of b-hadron production fractions in $7 \mathrm{TeV}$ pp collisions, Phys. Rev. D 85 (2012) 032008 [arXiv:1111.2357] [inSPIRE].

[15] LHCb collaboration, Measurement of the fragmentation fraction ratio $f_{s} / f_{d}$ and its dependence on B meson kinematics, JHEP 04 (2013) 001 [arXiv:1301.5286] [INSPIRE].

[16] LHCb collaboration, Updated average $f_{s} / f_{d}$ b-hadron production fraction ratio for $7 \mathrm{TeV} p p$ collisions, LHCb-CONF-2013-011 (2013).

[17] LHCb collaboration, The LHCb detector at the LHC, 2008 JINST 3 S08005 [INSPIRE].

[18] LHCb collaboration, LHCb detector performance, Int. J. Mod. Phys. A 30 (2015) 1530022 [arXiv: 1412.6352] [INSPIRE].

[19] T. Sjöstrand, S. Mrenna and P.Z. Skands, PYTHIA 6.4 physics and manual, JHEP 05 (2006) 026 [hep-ph/0603175] [INSPIRE].

[20] LHCb collaboration, Handling of the generation of primary events in Gauss, the LHCb simulation framework, J. Phys. Conf. Ser. 331 (2011) 032047 [InSPIRE].

[21] D.J. Lange, The EvtGen particle decay simulation package, Nucl. Instrum. Meth. A 462 (2001) 152 [INSPIRE].

[22] P. Golonka and Z. Was, PHOTOS Monte Carlo: a precision tool for QED corrections in Z and $W$ decays, Eur. Phys. J. C 45 (2006) 97 [hep-ph/0506026] [INSPIRE]. 
[23] Geant4 collaboration, J. Allison et al., GEANT4 developments and applications, IEEE Trans. Nucl. Sci. 53 (2006) 270.

[24] GEANT4 collaboration, S. Agostinelli et al., GEANT4: a simulation toolkit, Nucl. Instrum. Meth. A 506 (2003) 250 [inSPIRE].

[25] LHCb collaboration, The LHCb simulation application, Gauss: design, evolution and experience, J. Phys. Conf. Ser. 331 (2011) 032023 [inSPIRE].

[26] R. Aaij et al., The LHCb trigger and its performance in 2011, 2013 JINST 8 P04022 [arXiv: 1211.3055] [INSPIRE].

[27] V.V. Gligorov and M. Williams, Efficient, reliable and fast high-level triggering using a bonsai boosted decision tree, 2013 JINST 8 P02013 [arXiv:1210.6861] [INSPIRE].

[28] L. Breiman, J.H. Friedman, R.A. Olshen and C.J. Stone, Classification and regression trees, Wadsworth international group, Belmont U.S.A. (1984).

[29] R.E. Schapire and Y. Freund, A decision-theoretic generalization of on-line learning and an application to boosting, Jour. Comp. Syst. Sci. 55 (1997) 119.

[30] G. Punzi, Sensitivity of searches for new signals and its optimization, eConf C 030908 (2003) MODT002 [physics/0308063] [InSPIRE].

[31] M. Pivk and F.R. Le Diberder, SPlot: a statistical tool to unfold data distributions, Nucl. Instrum. Meth. A 555 (2005) 356 [physics/0402083] [INSPIRE].

[32] S. S. Wilks, The large-sample distribution of the likelihood ratio for testing composite hypotheses, Ann. Math.Statist. 9 (1938) 60.

[33] A. Martin Sanchez, CP violation studies on the $B^{0} \rightarrow D K^{* 0}$ decays and hadronic trigger performance with the LHCb detector at CERN, Ph.D. thesis, Universite Paris-sud XI, Paris, France (2013).

[34] I. Dunietz, R. Fleischer and U. Nierste, In pursuit of new physics with $B_{s}$ decays, Phys. Rev. D 63 (2001) 114015 [hep-ph/0012219].

[35] L. Lyons, D. Gibaut and P. Clifford, How to combine correlated estimates of a single physical quantity, Nucl. Instrum. Meth. A 270 (1988) 110 [INSPIRE].

[36] A. Valassi, Combining correlated measurements of several different physical quantities, Nucl. Instrum. Meth. A 500 (2003) 391 [INSPIRE].

[37] Particle Data Group collaboration, J. Beringer et al., Review of particle physics, Phys. Rev. D 86 (2012) 010001 [INSPIRE]. 


\section{The LHCb collaboration}

R. Aaij ${ }^{38}$, B. Adeva ${ }^{37}$, M. Adinolfi ${ }^{46}$, A. Affolder ${ }^{52}$, Z. Ajaltouni ${ }^{5}$, S. Akar ${ }^{6}$, J. Albrecht ${ }^{9}$, F. Alessio ${ }^{38}$, M. Alexander ${ }^{51}$, S. Ali ${ }^{41}$, G. Alkhazov ${ }^{30}$, P. Alvarez Cartelle ${ }^{53}$, A.A. Alves Jr ${ }^{57}$, S. Amato ${ }^{2}$, S. Amerio ${ }^{22}$, Y. Amhis ${ }^{7}$, L. An ${ }^{3}$, L. Anderlini ${ }^{17, g}$, J. Anderson ${ }^{40}$, M. Andreotti ${ }^{16, f}$, J.E. Andrews ${ }^{58}$, R.B. Appleby ${ }^{54}$, O. Aquines Gutierrez ${ }^{10}$, F. Archilli ${ }^{38}$, P. d'Argent ${ }^{11}$,

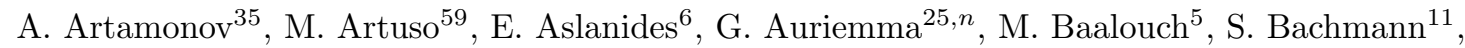
J.J. Back ${ }^{48}$, A. Badalov ${ }^{36}$, C. Baesso ${ }^{60}$, W. Baldini ${ }^{16,38}$, R.J. Barlow ${ }^{54}$, C. Barschel ${ }^{38}$, S. Barsuk ${ }^{7}$, W. Barter ${ }^{38}$, V. Batozskaya ${ }^{28}$, V. Battista ${ }^{39}$, A. Bay ${ }^{39}$, L. Beaucourt ${ }^{4}$, J. Beddow ${ }^{51}$, F. Bedeschi ${ }^{23}$, I. Bediaga ${ }^{1}$, L.J. Bel ${ }^{41}$, I. Belyaev ${ }^{31}$, E. Ben-Haim ${ }^{8}$, G. Bencivenni ${ }^{18}$, S. Benson ${ }^{38}$, J. Benton ${ }^{46}$, A. Berezhnoy ${ }^{32}$, R. Bernet ${ }^{40}$, A. Bertolin ${ }^{22}$, M.-O. Bettler ${ }^{38}$, M. van Beuzekom ${ }^{41}$, A. Bien ${ }^{11}$, S. Bifani ${ }^{45}$, T. Bird ${ }^{54}$, A. Birnkraut ${ }^{9}$, A. Bizzeti ${ }^{17, i}$, T. Blake ${ }^{48}$, F. Blanc ${ }^{39}$, J. Blouw $^{10}$, S. Blusk ${ }^{59}$, V. Bocci ${ }^{25}$, A. Bondar ${ }^{34}$, N. Bondar ${ }^{30,38}$, W. Bonivento ${ }^{15}$, S. Borghi ${ }^{54}$, M. Borsato ${ }^{7}$, T.J.V. Bowcock ${ }^{52}$, E. Bowen ${ }^{40}$, C. Bozzi ${ }^{16}$, S. Braun ${ }^{11}$, D. Brett ${ }^{54}$, M. Britsch ${ }^{10}$, T. Britton ${ }^{59}$, J. Brodzicka ${ }^{54}$, N.H. Brook ${ }^{46}$, A. Bursche ${ }^{40}$, J. Buytaert ${ }^{38}$, S. Cadeddu ${ }^{15}$, R. Calabrese ${ }^{16, f}$, M. Calvi ${ }^{20, k}$, M. Calvo Gomez ${ }^{36, p}$, P. Campana ${ }^{18}$, D. Campora Perez ${ }^{38}$, L. Capriotti ${ }^{54}$, A. Carbone ${ }^{14, d}$, G. Carboni ${ }^{24, l}$, R. Cardinale ${ }^{19, j}$, A. Cardini ${ }^{15}$, P. Carniti ${ }^{20}$, L. Carson ${ }^{50}$, K. Carvalho Akiba ${ }^{2,38}$, R. Casanova Mohr ${ }^{36}$, G. Casse ${ }^{52}$, L. Cassina ${ }^{20, k}$, L. Castillo Garcia ${ }^{38}$, M. Cattaneo ${ }^{38}$, Ch. Cauet ${ }^{9}$, G. Cavallero ${ }^{19}$, R. Cenci ${ }^{23, t}$, M. Charles ${ }^{8}$, Ph. Charpentier $^{38}$, M. Chefdeville ${ }^{4}$, S. Chen ${ }^{54}$, S.-F. Cheung ${ }^{55}$, N. Chiapolini ${ }^{40}$, M. Chrzaszcz ${ }^{40}$, X. Cid Vidal ${ }^{38}$, G. Ciezarek $^{41}$, P.E.L. Clarke ${ }^{50}$, M. Clemencic ${ }^{38}$, H.V. Cliff ${ }^{47}$, J. Closier ${ }^{38}$, V. Coco ${ }^{38}$, J. Cogan ${ }^{6}$,

E. Cogneras ${ }^{5}$, V. Cogoni ${ }^{15, e}$, L. Cojocariu ${ }^{29}$, G. Collazuol ${ }^{22}$, P. Collins ${ }^{38}$, A. Comerma-Montells ${ }^{11}$,

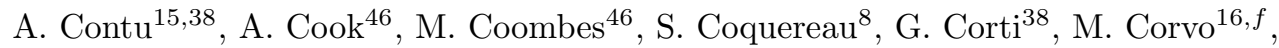
B. Couturier ${ }^{38}$, G.A. Cowan $^{50}$, D.C. Craik ${ }^{48}$, A. Crocombe ${ }^{48}$, M. Cruz Torres $^{60}$, S. Cunliffe ${ }^{53}$, R. Currie ${ }^{53}$, C. D'Ambrosio ${ }^{38}$, J. Dalseno ${ }^{46}$, P.N.Y. David ${ }^{41}$, A. Davis ${ }^{57}$, K. De Bruyn ${ }^{41}$, S. De Capua ${ }^{54}$, M. De Cian ${ }^{11}$, J.M. De Miranda ${ }^{1}$, L. De Paula ${ }^{2}$, W. De Silva ${ }^{57}$, P. De Simone ${ }^{18}$, C.-T. Dean ${ }^{51}$, D. Decamp ${ }^{4}$, M. Deckenhoff ${ }^{9}$, L. Del Buono ${ }^{8}$, N. Déléage ${ }^{4}$, D. Derkach ${ }^{55}$, O. Deschamps ${ }^{5}$, F. Dettori ${ }^{38}$, B. Dey ${ }^{40}$, A. Di Canto ${ }^{38}$, F. Di Ruscio ${ }^{24}$, H. Dijkstra ${ }^{38}$, S. Donleavy ${ }^{52}$, F. Dordei ${ }^{11}$, M. Dorigo ${ }^{39}$, A. Dosil Suárez ${ }^{37}$, D. Dossett ${ }^{48}$, A. Dovbnya ${ }^{43}$, K. Dreimanis ${ }^{52}$, L. Dufour ${ }^{41}$, G. Dujany ${ }^{54}$, F. Dupertuis ${ }^{39}$, P. Durante ${ }^{38}$, R. Dzhelyadin ${ }^{35}$, A. Dziurda ${ }^{26}$, A. Dzyuba ${ }^{30}$, S. Easo ${ }^{49,38}$, U. Egede ${ }^{53}$, V. Egorychev ${ }^{31}$, S. Eidelman ${ }^{34}$, S. Eisenhardt ${ }^{50}$, U. Eitschberger ${ }^{9}$, R. Ekelhof ${ }^{9}$, L. Eklund ${ }^{51}$, I. El Rifai ${ }^{5}$, Ch. Elsasser ${ }^{40}$, S. Ely ${ }^{59}$, S. Esen ${ }^{11}$, H.M. Evans ${ }^{47}$, T. Evans ${ }^{55}$, A. Falabella ${ }^{14}$, C. Färber ${ }^{11}$, C. Farinelli ${ }^{41}$, N. Farley ${ }^{45}$, S. Farry ${ }^{52}$, R. Fay ${ }^{52}$, D. Ferguson ${ }^{50}$, V. Fernandez Albor ${ }^{37}$, F. Ferrari ${ }^{14}$, F. Ferreira Rodrigues ${ }^{1}$, M. Ferro-Luzzi ${ }^{38}$, S. Filippov ${ }^{33}$, M. Fiore ${ }^{16,38, f}$, M. Fiorini ${ }^{16, f}$, M. Firlej ${ }^{27}$, C. Fitzpatrick ${ }^{39}$, T. Fiutowski ${ }^{27}$, K. Fohl ${ }^{38}$, P. Fol ${ }^{53}$, M. Fontana ${ }^{10}$, F. Fontanelli ${ }^{19, j}$, R. Forty ${ }^{38}$, O. Francisco ${ }^{2}$, M. Frank ${ }^{38}$, C. Frei ${ }^{38}$, M. Frosini ${ }^{17}$, J. Fu ${ }^{21}$, E. Furfaro ${ }^{24, l}$, A. Gallas Torreira ${ }^{37}$, D. Galli ${ }^{14, d}$, S. Gallorini ${ }^{22,38}$, S. Gambetta ${ }^{50}$, M. Gandelman ${ }^{2}$, P. Gandini ${ }^{55}$, Y. Gao ${ }^{3}$, J. García Pardiñas ${ }^{37}$, J. Garofoli ${ }^{59}$, J. Garra Tico ${ }^{47}$, L. Garrido ${ }^{36}$, D. Gascon ${ }^{36}$, C. Gaspar ${ }^{38}$, R. Gauld ${ }^{55}$, L. Gavardi ${ }^{9}$, G. Gazzoni ${ }^{5}$, A. Geraci ${ }^{21, v}$, D. Gerick ${ }^{11}$, E. Gersabeck ${ }^{11}$, M. Gersabeck ${ }^{54}$, T. Gershon ${ }^{48}$, Ph. Ghez ${ }^{4}$, A. Gianelle ${ }^{22}$, S. Gianì ${ }^{39}$, V. Gibson ${ }^{47}$, O. G. Girard ${ }^{39}$, L. Giubega ${ }^{29}$, V.V. Gligorov ${ }^{38}$, C. Göbel ${ }^{60}$, D. Golubkov ${ }^{31}$, A. Golutvin ${ }^{53,31,38}$, A. Gomes ${ }^{1, a}$, C. Gotti $^{20, k}$,

M. Grabalosa Gándara ${ }^{5}$, R. Graciani Diaz ${ }^{36}$, L.A. Granado Cardoso ${ }^{38}$, E. Graugés ${ }^{36}$, E. Graverini ${ }^{40}$, G. Graziani ${ }^{17}$, A. Grecu ${ }^{29}$, E. Greening ${ }^{55}$, S. Gregson ${ }^{47}$, P. Griffith ${ }^{45}$, L. Grillo ${ }^{11}$, O. Grünberg ${ }^{63}$, B. Gui ${ }^{59}$, E. Gushchin ${ }^{33}$, Yu. Guz ${ }^{35,38}$, T. Gys ${ }^{38}$, C. Hadjivasiliou ${ }^{59}$, G. Haefeli ${ }^{39}$,

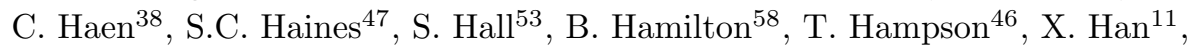

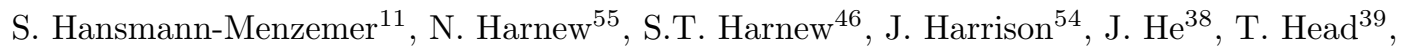
V. Heijne ${ }^{41}$, K. Hennessy ${ }^{52}$, P. Henrard ${ }^{5}$, L. Henry ${ }^{8}$, J.A. Hernando Morata ${ }^{37}$, 
E. van Herwijnen ${ }^{38}$, M. Heß ${ }^{63}$, A. Hicheur ${ }^{2}$, D. Hill ${ }^{55}$, M. Hoballah ${ }^{5}$, C. Hombach ${ }^{54}$, W. Hulsbergen ${ }^{41}$, T. Humair ${ }^{53}$, N. Hussain ${ }^{55}$, D. Hutchcroft ${ }^{52}$, D. Hynds ${ }^{51}$, M. Idzik ${ }^{27}$, P. Ilten ${ }^{56}$, R. Jacobsson ${ }^{38}$, A. Jaeger ${ }^{11}$, J. Jalocha ${ }^{55}$, E. Jans ${ }^{41}$, A. Jawahery ${ }^{58}$, F. Jing ${ }^{3}$, M. John ${ }^{55}$, D. Johnson ${ }^{38}$, C.R. Jones ${ }^{47}$, C. Joram ${ }^{38}$, B. Jost ${ }^{38}$, N. Jurik ${ }^{59}$, S. Kandybei ${ }^{43}$, W. Kanso ${ }^{6}$, M. Karacson ${ }^{38}$, T.M. Karbach ${ }^{38, \dagger}$, S. Karodia ${ }^{51}$, M. Kelsey ${ }^{59}$, I.R. Kenyon ${ }^{45}$, M. Kenzie ${ }^{38}$, T. Ketel ${ }^{42}$, B. Khanji ${ }^{20,38, k}$, C. Khurewathanakul ${ }^{39}$, S. Klaver ${ }^{54}$, K. Klimaszewski ${ }^{28}$, O. Kochebina ${ }^{7}$, M. Kolpin ${ }^{11}$, I. Komarov ${ }^{39}$, R.F. Koopman ${ }^{42}$, P. Koppenburg ${ }^{41,38}$, L. Kravchuk ${ }^{33}$, K. Kreplin ${ }^{11}$, M. Kreps ${ }^{48}$, G. Krocker ${ }^{11}$, P. Krokovny ${ }^{34}$, F. Kruse ${ }^{9}$, W. Kucewicz ${ }^{26, o}$, M. Kucharczyk ${ }^{26}$, V. Kudryavtsev ${ }^{34}$, A. K. Kuonen ${ }^{39}$, K. Kurek ${ }^{28}$, T. Kvaratskheliya ${ }^{31}$, V.N. La Thi ${ }^{39}$, D. Lacarrere ${ }^{38}$, G. Lafferty ${ }^{54}$, A. Lai ${ }^{15}$, D. Lambert ${ }^{50}$, R.W. Lambert ${ }^{42}$, G. Lanfranchi ${ }^{18}$, C. Langenbruch ${ }^{48}$, B. Langhans ${ }^{38}$, T. Latham ${ }^{48}$, C. Lazzeroni ${ }^{45}$, R. Le Gac ${ }^{6}$, J. van Leerdam ${ }^{41}$, J.-P. Lees ${ }^{4}$, R. Lefèvre ${ }^{5}$, A. Leflat ${ }^{32,38}$, J. Lefrançois ${ }^{7}$, O. Leroy ${ }^{6}$, T. Lesiak ${ }^{26}$,

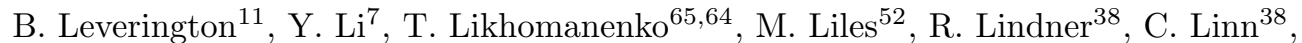

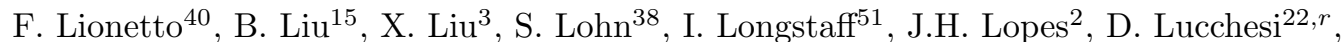
M. Lucio Martinez ${ }^{37}$, H. Luo ${ }^{50}$, A. Lupato ${ }^{22}$, E. Luppi ${ }^{16, f}$, O. Lupton ${ }^{55}$, F. Machefert ${ }^{7}$,

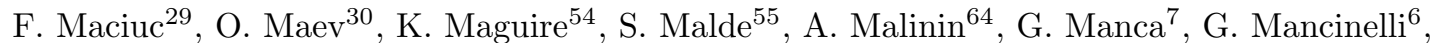
P. Manning ${ }^{59}$, A. Mapelli ${ }^{38}$, J. Maratas ${ }^{5}$, J.F. Marchand ${ }^{4}$, U. Marconi ${ }^{14}$, C. Marin Benito ${ }^{36}$, P. Marino ${ }^{23,38, t}$, R. Märki ${ }^{39}$, J. Marks ${ }^{11}$, G. Martellotti ${ }^{25}$, M. Martinelli ${ }^{39}$, D. Martinez Santos ${ }^{42}$, F. Martinez Vidal ${ }^{66}$, D. Martins Tostes ${ }^{2}$, A. Massafferri ${ }^{1}$, R. Matev ${ }^{38}$, A. Mathad $^{48}$, Z. Mathe $^{38}$, C. Matteuzzi ${ }^{20}$, K. Matthieu ${ }^{11}$, A. Mauri ${ }^{40}$, B. Maurin ${ }^{39}$, A. Mazurov ${ }^{45}$, M. McCann ${ }^{53}$, J. McCarthy ${ }^{45}$, A. McNab ${ }^{54}$, R. McNulty ${ }^{12}$, B. Meadows ${ }^{57}$, F. Meier ${ }^{9}$, M. Meissner ${ }^{11}$, M. Merk ${ }^{41}$, D.A. Milanes ${ }^{62}$, M.-N. Minard ${ }^{4}$, D.S. Mitzel ${ }^{11}$, J. Molina Rodriguez ${ }^{60}$, S. Monteil ${ }^{5}$, M. Morandin ${ }^{22}$, P. Morawski27 ${ }^{27}$, A. Mordà ${ }^{6}$, M.J. Morello ${ }^{23, t}$, J. Moron ${ }^{27}$, A.B. Morris ${ }^{50}$, R. Mountain ${ }^{59}$, F. Muheim ${ }^{50}$, J. Müller ${ }^{9}$, K. Müller ${ }^{40}$, V. Müller ${ }^{9}$, M. Mussini ${ }^{14}$, B. Muster ${ }^{39}$, P. Naik ${ }^{46}$, T. Nakada ${ }^{39}$, R. Nandakumar ${ }^{49}$, I. Nasteva ${ }^{2}$, M. Needham ${ }^{50}$, N. Neri ${ }^{21}$, S. Neubert ${ }^{11}$, N. Neufeld ${ }^{38}$, M. Neuner ${ }^{11}$, A.D. Nguyen ${ }^{39}$, T.D. Nguyen ${ }^{39}$, C. Nguyen-Mau ${ }^{39, q}$, V. Niess ${ }^{5}$, R. Niet ${ }^{9}$, N. Nikitin ${ }^{32}$, T. Nikodem ${ }^{11}$, D. Ninci' ${ }^{23}$, A. Novoselov ${ }^{35}$, D.P. O'Hanlon ${ }^{48}$, A. Oblakowska-Mucha ${ }^{27}$, V. Obraztsov ${ }^{35}$, S. Ogilvy ${ }^{51}$, O. Okhrimenko ${ }^{44}$, R. Oldeman ${ }^{15, e}$, C.J.G. Onderwater ${ }^{67}$, B. Osorio Rodrigues ${ }^{1}$, J.M. Otalora Goicochea ${ }^{2}$, A. Otto ${ }^{38}$, P. Owen ${ }^{53}$, A. Oyanguren ${ }^{66}$, A. Palano ${ }^{13, c}$, F. Palombo ${ }^{21, u}$, M. Palutan ${ }^{18}$, J. Panman $^{38}$, A. Papanestis ${ }^{49}$, M. Pappagallo ${ }^{51}$, L.L. Pappalardo ${ }^{16, f}$, C. Parkes ${ }^{54}$, G. Passaleva ${ }^{17}$, G.D. Patel ${ }^{52}$, M. Patel ${ }^{53}$, C. Patrignani ${ }^{19, j}$, A. Pearce ${ }^{54,49}$, A. Pellegrino ${ }^{41}$, G. Penso ${ }^{25, m}$, M. Pepe Altarelli ${ }^{38}$, S. Perazzini ${ }^{14, d}$, P. Perret ${ }^{5}$, L. Pescatore ${ }^{45}$, K. Petridis ${ }^{46}$, A. Petrolini ${ }^{19, j}$, E. Picatoste Olloqui ${ }^{36}$, B. Pietrzyk ${ }^{4}$, T. Pilař ${ }^{48}$, D. Pinci ${ }^{25}$, A. Pistone ${ }^{19}$, A. Piucci ${ }^{11}$, S. Playfer ${ }^{50}$, M. Plo Casasus ${ }^{37}$, T. Poikela ${ }^{38}$, F. Polci ${ }^{8}$, A. Poluektov ${ }^{48,34}$, I. Polyakov ${ }^{31}$, E. Polycarpo ${ }^{2}$, A. Popov ${ }^{35}$, D. Popov ${ }^{10,38}$, B. Popovici ${ }^{29}$, C. Potterat ${ }^{2}$, E. Price ${ }^{46}$, J.D. Price ${ }^{52}$, J. Prisciandaro ${ }^{39}$, A. Pritchard ${ }^{52}$, C. Prouve ${ }^{46}$, V. Pugatch ${ }^{44}$, A. Puig Navarro ${ }^{39}$, G. Punzi ${ }^{23, s}$, W. Qian ${ }^{4}$, R. Quagliani ${ }^{7,46}$, B. Rachwal ${ }^{26}$, J.H. Rademacker ${ }^{46}$, B. Rakotomiaramanana ${ }^{39}$, M. Rama ${ }^{23}$, M.S. Rangel ${ }^{2}$, I. Raniuk ${ }^{43}$, N. Rauschmayr ${ }^{38}$, G. Raven ${ }^{42}$, F. Redi ${ }^{53}$, S. Reichert ${ }^{54}$, M.M. Reid ${ }^{48}$, A.C. dos Reis ${ }^{1}$, S. Ricciardi ${ }^{49}$, S. Richards ${ }^{46}$, M. Rihl ${ }^{38}$, K. Rinnert ${ }^{52}$, V. Rives Molina ${ }^{36}$, P. Robbe ${ }^{7,38}$, A.B. Rodrigues ${ }^{1}$, E. Rodrigues ${ }^{54}$, J.A. Rodriguez Lopez ${ }^{62}$, P. Rodriguez Perez ${ }^{54}$, S. Roiser ${ }^{38}$, V. Romanovsky ${ }^{35}$, A. Romero Vidal ${ }^{37}$, M. Rotondo ${ }^{22}$, J. Rouvinet ${ }^{39}$, T. Ruf ${ }^{38}$, H. Ruiz ${ }^{36}$, P. Ruiz Valls ${ }^{66}$, J.J. Saborido Silva ${ }^{37}$, N. Sagidova ${ }^{30}$, P. Sail ${ }^{51}$, B. Saitta ${ }^{15, e}$, V. Salustino Guimaraes ${ }^{2}$, C. Sanchez Mayordomo ${ }^{66}$, B. Sanmartin Sedes ${ }^{37}$, R. Santacesaria ${ }^{25}$, C. Santamarina Rios ${ }^{37}$, M. Santimaria ${ }^{18}$, E. Santovetti ${ }^{24, l}$, A. Sarti ${ }^{18, m}$, C. Satriano ${ }^{25, n}$, A. Satta ${ }^{24}$, D.M. Saunders ${ }^{46}$, D. Savrina ${ }^{31,32}$, M. Schiller ${ }^{38}$, H. Schindler ${ }^{38}$, M. Schlupp ${ }^{9}$, M. Schmelling ${ }^{10}$, T. Schmelzer ${ }^{9}$, B. Schmidt ${ }^{38}$, O. Schneider ${ }^{39}$, A. Schopper ${ }^{38}$, M. Schubiger ${ }^{39}$, M.-H. Schune ${ }^{7}$, R. Schwemmer ${ }^{38}$, B. Sciascia ${ }^{18}$, A. Sciubba ${ }^{25, m}$, A. Semennikov ${ }^{31}$, I. Sepp ${ }^{53}$, 
N. Serra ${ }^{40}$, J. Serrano ${ }^{6}$, L. Sestini ${ }^{22}$, P. Seyfert ${ }^{11}$, M. Shapkin ${ }^{35}$, I. Shapoval ${ }^{16,43, f}$,

Y. Shcheglov ${ }^{30}$, T. Shears ${ }^{52}$, L. Shekhtman ${ }^{34}$, V. Shevchenko ${ }^{64}$, A. Shires ${ }^{9}$, R. Silva Coutinho ${ }^{48}$,

G. Simi ${ }^{22}$, M. Sirendi ${ }^{47}$, N. Skidmore ${ }^{46}$, I. Skillicorn ${ }^{51}$, T. Skwarnicki ${ }^{59}$, E. Smith ${ }^{55,49}$, E. Smith ${ }^{53}$,

I. T. Smith ${ }^{50}$, J. Smith ${ }^{47}$, M. Smith ${ }^{54}$, H. Snoek ${ }^{41}$, M.D. Sokoloff ${ }^{57,38}$, F.J.P. Soler ${ }^{51}$,

F. Soomro ${ }^{39}$, D. Souza ${ }^{46}$, B. Souza De Paula ${ }^{2}$, B. Spaan ${ }^{9}$, P. Spradlin ${ }^{51}$, S. Sridharan ${ }^{38}$,

F. Stagni ${ }^{38}$, M. Stahl ${ }^{11}$, S. Stahl ${ }^{38}$, O. Steinkamp ${ }^{40}$, O. Stenyakin ${ }^{35}$, F. Sterpka ${ }^{59}$, S. Stevenson ${ }^{55}$,

S. Stoica ${ }^{29}$, S. Stone ${ }^{59}$, B. Storaci ${ }^{40}$, S. Stracka ${ }^{23, t}$, M. Straticiuc ${ }^{29}$, U. Straumann ${ }^{40}$, L. Sun ${ }^{57}$,

W. Sutcliffe ${ }^{53}$, K. Swientek ${ }^{27}$, S. Swientek ${ }^{9}$, V. Syropoulos ${ }^{42}$, M. Szczekowski ${ }^{28}$, P. Szczypka ${ }^{39,38}$,

T. Szumlak ${ }^{27}$, S. T'Jampens ${ }^{4}$, T. Tekampe ${ }^{9}$, M. Teklishyn ${ }^{7}$, G. Tellarini ${ }^{16, f}$, F. Teubert ${ }^{38}$,

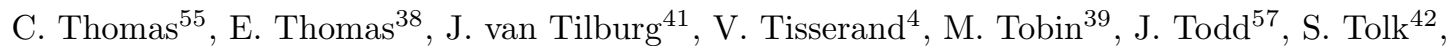

L. Tomassetti ${ }^{16, f}$, D. Tonelli ${ }^{38}$, S. Topp-Joergensen ${ }^{55}$, N. Torr ${ }^{55}$, E. Tournefier ${ }^{4}$, S. Tourneur ${ }^{39}$,

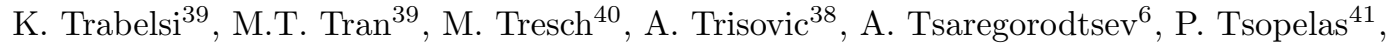

N. Tuning ${ }^{41,38}$, A. Ukleja ${ }^{28}$, A. Ustyuzhanin ${ }^{65,64}$, U. Uwer ${ }^{11}$, C. Vacca ${ }^{15, e}$, V. Vagnoni ${ }^{14}$,

G. Valenti ${ }^{14}$, A. Vallier ${ }^{7}$, R. Vazquez Gomez ${ }^{18}$, P. Vazquez Regueiro ${ }^{37}$, C. Vázquez Sierra ${ }^{37}$,

S. Vecchi ${ }^{16}$, J.J. Velthuis ${ }^{46}$, M. Veltri ${ }^{17, h}$, G. Veneziano ${ }^{39}$, M. Vesterinen ${ }^{11}$, B. Viaud ${ }^{7}$, D. Vieira ${ }^{2}$, M. Vieites Diaz ${ }^{37}$, X. Vilasis-Cardona ${ }^{36, p}$, A. Vollhardt ${ }^{40}$, D. Volyanskyy ${ }^{10}$, D. Voong ${ }^{46}$,

A. Vorobyev ${ }^{30}$, V. Vorobyev ${ }^{34}$, C. Voß ${ }^{63}$, J.A. de Vries ${ }^{41}$, R. Waldi ${ }^{63}$, C. Wallace ${ }^{48}$, R. Wallace ${ }^{12}$, J. Walsh ${ }^{23}$, S. Wandernoth ${ }^{11}$, J. Wang ${ }^{59}$, D.R. Ward ${ }^{47}$, N.K. Watson ${ }^{45}$, D. Websdale ${ }^{53}$,

A. Weiden ${ }^{40}$, M. Whitehead ${ }^{48}$, D. Wiedner ${ }^{11}$, G. Wilkinson ${ }^{55,38}$, M. Wilkinson ${ }^{59}$, M. Williams $^{38}$, M.P. Williams ${ }^{45}$, M. Williams ${ }^{56}$, T. Williams ${ }^{45}$, F.F. Wilson ${ }^{49}$, J. Wimberley ${ }^{58}$, J. Wishahi ${ }^{9}$, W. Wislicki ${ }^{28}$, M. Witek ${ }^{26}$, G. Wormser ${ }^{7}$, S.A. Wotton ${ }^{47}$, S. Wright ${ }^{47}$, K. Wyllie ${ }^{38}$, Y. Xie ${ }^{61}$, Z. Xu ${ }^{39}$, Z. Yang ${ }^{3}$, J. Yu ${ }^{61}$, X. Yuan ${ }^{34}$, O. Yushchenko ${ }^{35}$, M. Zangoli ${ }^{14}$, M. Zavertyaev ${ }^{10, b}$,

L. Zhang ${ }^{3}$, Y. Zhang ${ }^{3}$, A. Zhelezov ${ }^{11}$, A. Zhokhov ${ }^{31}$, L. Zhong ${ }^{3}$

1 Centro Brasileiro de Pesquisas Físicas (CBPF), Rio de Janeiro, Brazil

2 Universidade Federal do Rio de Janeiro (UFRJ), Rio de Janeiro, Brazil

3 Center for High Energy Physics, Tsinghua University, Beijing, China

4 LAPP, Université Savoie Mont-Blanc, CNRS/IN2P3, Annecy-Le-Vieux, France

5 Clermont Université, Université Blaise Pascal, CNRS/IN2P3, LPC, Clermont-Ferrand, France

6 CPPM, Aix-Marseille Université, CNRS/IN2P3, Marseille, France

7 LAL, Université Paris-Sud, CNRS/IN2P3, Orsay, France

8 LPNHE, Université Pierre et Marie Curie, Université Paris Diderot, CNRS/IN2P3, Paris, France

9 Fakultät Physik, Technische Universität Dortmund, Dortmund, Germany

10 Max-Planck-Institut für Kernphysik (MPIK), Heidelberg, Germany

11 Physikalisches Institut, Ruprecht-Karls-Universität Heidelberg, Heidelberg, Germany

12 School of Physics, University College Dublin, Dublin, Ireland

13 Sezione INFN di Bari, Bari, Italy

14 Sezione INFN di Bologna, Bologna, Italy

15 Sezione INFN di Cagliari, Cagliari, Italy

16 Sezione INFN di Ferrara, Ferrara, Italy

17 Sezione INFN di Firenze, Firenze, Italy

18 Laboratori Nazionali dell'INFN di Frascati, Frascati, Italy

19 Sezione INFN di Genova, Genova, Italy

20 Sezione INFN di Milano Bicocca, Milano, Italy

21 Sezione INFN di Milano, Milano, Italy

22 Sezione INFN di Padova, Padova, Italy

23 Sezione INFN di Pisa, Pisa, Italy

24 Sezione INFN di Roma Tor Vergata, Roma, Italy

25 Sezione INFN di Roma La Sapienza, Roma, Italy

26 Henryk Niewodniczanski Institute of Nuclear Physics Polish Academy of Sciences, Kraków, Poland 
AGH - University of Science and Technology, Faculty of Physics and Applied Computer Science, Kraków, Poland

28 National Center for Nuclear Research (NCBJ), Warsaw, Poland

29 Horia Hulubei National Institute of Physics and Nuclear Engineering, Bucharest-Magurele, Romania

30 Petersburg Nuclear Physics Institute (PNPI), Gatchina, Russia

31 Institute of Theoretical and Experimental Physics (ITEP), Moscow, Russia

32 Institute of Nuclear Physics, Moscow State University (SINP MSU), Moscow, Russia

33 Institute for Nuclear Research of the Russian Academy of Sciences (INR RAN), Moscow, Russia

34 Budker Institute of Nuclear Physics (SB RAS) and Novosibirsk State University, Novosibirsk, Russia

35 Institute for High Energy Physics (IHEP), Protvino, Russia

36 Universitat de Barcelona, Barcelona, Spain

37 Universidad de Santiago de Compostela, Santiago de Compostela, Spain

38 European Organization for Nuclear Research (CERN), Geneva, Switzerland

39 Ecole Polytechnique Fédérale de Lausanne (EPFL), Lausanne, Switzerland

40 Physik-Institut, Universität Zürich, Zürich, Switzerland

41 Nikhef National Institute for Subatomic Physics, Amsterdam, The Netherlands

42 Nikhef National Institute for Subatomic Physics and VU University Amsterdam, Amsterdam, The Netherlands

43 NSC Kharkiv Institute of Physics and Technology (NSC KIPT), Kharkiv, Ukraine

44 Institute for Nuclear Research of the National Academy of Sciences (KINR), Kyiv, Ukraine

45 University of Birmingham, Birmingham, United Kingdom

46 H.H. Wills Physics Laboratory, University of Bristol, Bristol, United Kingdom

47 Cavendish Laboratory, University of Cambridge, Cambridge, United Kingdom

48 Department of Physics, University of Warwick, Coventry, United Kingdom

49 STFC Rutherford Appleton Laboratory, Didcot, United Kingdom

50 School of Physics and Astronomy, University of Edinburgh, Edinburgh, United Kingdom

51 School of Physics and Astronomy, University of Glasgow, Glasgow, United Kingdom

52 Oliver Lodge Laboratory, University of Liverpool, Liverpool, United Kingdom

53 Imperial College London, London, United Kingdom

54 School of Physics and Astronomy, University of Manchester, Manchester, United Kingdom

55 Department of Physics, University of Oxford, Oxford, United Kingdom

56 Massachusetts Institute of Technology, Cambridge, MA, United States

57 University of Cincinnati, Cincinnati, OH, United States

58 University of Maryland, College Park, MD, United States

59 Syracuse University, Syracuse, NY, United States

60 Pontifícia Universidade Católica do Rio de Janeiro (PUC-Rio), Rio de Janeiro, Brazil, associated $\mathrm{o}^{2}$

61 Institute of Particle Physics, Central China Normal University, Wuhan, Hubei, China, associated to $^{3}$

62 Departamento de Fisica, Universidad Nacional de Colombia, Bogota, Colombia, associated to ${ }^{8}$

63 Institut für Physik, Universität Rostock, Rostock, Germany, associated to ${ }^{11}$

64 National Research Centre Kurchatov Institute, Moscow, Russia, associated to ${ }^{31}$

65 Yandex School of Data Analysis, Moscow, Russia, associated to ${ }^{31}$

66 Instituto de Fisica Corpuscular (IFIC), Universitat de Valencia-CSIC, Valencia, Spain, associated $t^{36}$

67 Van Swinderen Institute, University of Groningen, Groningen, The Netherlands, associated to ${ }^{41}$

a Universidade Federal do Triângulo Mineiro (UFTM), Uberaba-MG, Brazil

${ }^{b}$ P.N. Lebedev Physical Institute, Russian Academy of Science (LPI RAS), Moscow, Russia

c Università di Bari, Bari, Italy 
d Università di Bologna, Bologna, Italy

e Università di Cagliari, Cagliari, Italy

$f$ Università di Ferrara, Ferrara, Italy

$g$ Università di Firenze, Firenze, Italy

$h$ Università di Urbino, Urbino, Italy

i Università di Modena e Reggio Emilia, Modena, Italy

j Università di Genova, Genova, Italy

* Università di Milano Bicocca, Milano, Italy

$l$ Università di Roma Tor Vergata, Roma, Italy

$m$ Università di Roma La Sapienza, Roma, Italy

$n$ Università della Basilicata, Potenza, Italy

- AGH - University of Science and Technology, Faculty of Computer Science, Electronics and Telecommunications, Kraków, Poland

$p$ LIFAELS, La Salle, Universitat Ramon Llull, Barcelona, Spain

$q$ Hanoi University of Science, Hanoi, Viet Nam

$r$ Università di Padova, Padova, Italy

$s$ Università di Pisa, Pisa, Italy

${ }^{t}$ Scuola Normale Superiore, Pisa, Italy

u Università degli Studi di Milano, Milano, Italy

$\checkmark$ Politecnico di Milano, Milano, Italy ${ }^{\dagger}$ Deceased 